\title{
La communication organisationnelle au sein d'une entreprise de presse écrite
}

Audrey de Ceglie

\section{(2) OpenEdition}

1 Journals

Édition électronique

URL : http://journals.openedition.org/communicationorganisation/204

DOI : 10.4000/communicationorganisation.204

ISSN : $1775-3546$

Éditeur

Presses universitaires de Bordeaux

Édition imprimée

Date de publication : 1 juillet 2007

Pagination : 196-213

ISSN : 1168-5549

\section{Référence électronique}

Audrey de Ceglie, "La communication organisationnelle au sein d'une entreprise de presse écrite »,

Communication et organisation [En ligne], 31 | 2007, mis en ligne le 01 juillet 2010, consulté le 19 avril 2019. URL : http://journals.openedition.org/communicationorganisation/204 ; DOI : 10.4000/

communicationorganisation.204 


\section{Expériences}

\section{Résumé}

L'objet de cet article est d'étudier un organisme de presse comme un système artefactuel, c'est-à-dire une construction de la réalité à un moment donné. Pour cela, l'unité de base de cette étude est le geste professionnel d'écriture des journalistes. Ce dernier sera analysé comme un processus de médiation, c'est-à-dire en observant comment les processus de communication et d'interaction via le journal se construisent, mais aussi comme un processus de travail collaboratif. $\mathrm{Au}$ quotidien, l'organisation journalistique produit de l'information à partir de données brutes de terrain. Elle permet au lecteur de comprendre le monde qui l'entoure. Pour nous, l'information n'est pas le fruit d'un seul acteur, le journaliste. C'est un produit collectif relevant d'un sens commun dans un contexte spécifique.

Mots clés : Entreprises de presse, journalistes, médiation journalistique, construction sociale de la réalité.

\section{Abstract}

This research analyses a press enterprise as an "artefact », namely a construction of reality. Journalist's work is in fact a mediation process: data are picked in the social life, and are re-built linked with the choice of subjects and the writing of press articles. It's a collaborative work, which designs a specific picture of reality : it can influence the reader's understanding of the world. Press information isn't only the production of journalists, but is also the result of a social construction.

Keywords : press enterprises, journalist works, mediation process, social construction of reality.

Audrey de Ceglie est doctorante en Sciences de l'Information et de la Communication au Centre de Recherche et d'Analyse en Information et Communication (CRAIC - Jeune Equipe $\mathrm{n}^{\circ} 2469$ ) de l'Université Aix-Marseille 3. Ses recherches portent sur le fonctionnement des entreprises de presse et le travail collaboratif des journalistes, appréhendé comme un processus de médiation. 


\section{La communication organisationnelle au sein d'une entreprise de presse écrite Audrey de Ceglie}

deceglie@hotmail.com

La communication organisationnelle intéresse, depuis la seconde moitié du $\mathrm{XX}^{\mathrm{e}}$ siècle, de nombreux champs de recherche allant de la Sociologie à l'Anthropologie, à la Psychologie, et aux Sciences de l'Administration (Caron, 2004). En Sciences de l'Information et de la Communication, la communication organisationnelle devient l'objet central de nombreuses recherches qui s'intéressent aux débats théoriques sur les organisations et plus particulièrement aux processus de communication et aux moyens mis en œuvre pour élaborer des activités orientées vers un but (Bouzon \& Meyer, 2006).

L'objet de cet article est d'étudier un organisme de presse comme un système artefactuel, c'est-à-dire une construction de la réalité à un moment donné. Pour cela, l'unité de base de cette étude est le geste professionnel d'écriture des journalistes. Ce dernier sera analysé comme un processus de médiation, c'est-à-dire en observant comment les processus de communication et d'interaction via le journal se construisent, mais aussi comme un processus de travail collaboratif. Au quotidien, l'organisation journalistique produit de l'information à partir de données brutes de terrain. Elle permet au lecteur de comprendre le monde qui l'entoure. Cette information est un construit social, à un moment donné, qui véhicule une construction collective de la réalité sociale. Pour nous, l'information n'est pas le fruit d'un seul acteur, le journaliste. C'est un produit collectif relevant d'un sens commun dans un contexte spécifique.

Suite à une analyse de terrain effectuée dans un organisme de presse quotidienne régionale, nous avons étudié comment les processus de médiation de l'information sont à l'origine de l'élaboration du geste professionnel des journalistes lors de la rédaction et comment l'organisation journalistique relève de situations de coopération où les processus communicationnels peuvent expliquer leur élaboration.

Le but de cet article est de montrer que les organismes de presse écrite ne sont pas uniquement un lieu de médiatisation de l'information, c'est-à-dire de transposition d'un code linguistique, visuel... en un autre code pour le lecteur, comme c'est souvent le cas dans les recherches sur la presse; mais plus un lieu de médiation de l'information, c'est-à-dire d'interactions et de communications entre 


\section{Expériences}

acteurs via un journal. Notre article va dans la continuité des travaux de recherche de Bruno David $(2002$; 2004) sur la photographie de presse mais en nous attachant plus particulièrement au geste professionnel d'écriture. Cet auteur montre que la photographie de presse au sein des rédactions journalistiques résulte d'un travail collectif des acteurs : "la signification de la photographie procède d'une lente écriture collective » (David, 2002, p. 72).

Ici, la communication organisationnelle sera entendue comme "un processus complexe dans lequel la signification des messages n'est pas une donnée a priori mais une construction culturellement marquée qui s'élabore collectivement selon les situations, dans le temps et dans l'espace» (Bouzon \& Meyer, 2006, p. 6). Notre point de vue n'envisage donc pas la presse écrite comme une simple organisation, définie comme «un ensemble de moyens tenus ensemble par des règles et rassemblée autour d'un but qui la contraint et oriente ces activités » (Guyot, 2004, p. 1) mais, comme le produit d'une activité humaine socialement organisée qui déclenche une activité intellectuelle élaborant des processus nécessaires à la création d'un produit : le journal. La presse se situe au niveau de l'analyse de la mise au travail ou de l'organisation du travail, c'est-à-dire de l'utilisation de force productive et des relations et des interactions entre les acteurs comme vecteur de l'activité (Le Moënne, 2006, p. 17). C'est par une approche artefactuelle du système médiatique que nous observons les médiations entre les acteurs comme des constructions sociales de la réalité où l'article devient indissociable des conditions de sa réalisation, du contexte, des acteurs...

Les informations que construisent les journalistes sont subjectives et relèvent de l'expérience, des interactions dans un contexte donné, mais aussi de la collaboration qui anime le travail de rédaction. Chaque journaliste a ses propres modes de pensée, ses propres informations et connaissances, ainsi que ses propres représentations, mais c'est dans l'interaction qu'il va leur donner sens. Les informations se construisent alors progressivement, de manière individuelle et collective, sollicitant des capacités cognitives importantes qui prennent leur origine dans la médiation.

Grâce au travail coopératif entre les divers acteurs du système journalistique (rédacteurs en chef, responsables de la pagination, rédacteurs, secrétaires de rédaction, documentalistes, photographes etc., mais aussi les lecteurs) se construit l'information journalistique. En effet, l'information journalistique est le fruit d'une construction collective de l'information entre les journalistes grâce aux 
La communication organisationnelle au sein d'une entreprise de

presse écrite

communications et interactions au sein du système journalistique et grâce aux interactions et relations médiées par le journal, que les rédacteurs entretiennent avec les lecteurs. L'information journalistique dans notre étude est perçue comme un pré-construit permettant aux lecteurs de construire leur propre perception de la réalité au moment de la publication. Les actions des journalistes sont étudiées dans cet article, au sein d'un contexte que l'on considère comme indexical et récursif, c'est-à-dire qu'il agit sur les interactions sociales, tout en évoluant grâce à elles. Cette approche permet de comprendre comment l'information véhiculée apparemment par l'image d'un seul individu est en réalité le résultat d'un travail collectif. Nous étudions comment la presse écrite peut être perçue comme un processus de médiation de l'information. Pour cela, nous montrons que les processus de médiation de l'information sont le résultat d'un processus de communication, d'interaction et de relations entre acteurs (journalistes, lecteurs...), mais aussi un processus de construction collective d'un geste professionnel d'écriture.

Nous étudions, d'une part les processus communicationnels, grâce aux travaux de Jean-Luc Bouillon (2003) sur les dimensions communicationnelles, pour montrer que les interactions verbales ont lieu dans un contexte particulier; d'autre part, les processus de médiation comme le fruit d'une information située (Lave, 1988), distribuée (Hutchins, 1995) et contextuellement partagée (Salembier \& Zouinar, 2004) s'appuient sur les recherches menées par Arlette Bouzon (2003) et Brigitte Guyot (2004) sur les activités coopératives.

Nous envisageons, donc, l'étude du geste professionnel d'écriture du journaliste comme un phénomène social et culturel révélateur d'une action située et d'une connaissance partagée. Ce point de vue vient en complément des approches disciplinaires des phénomènes d'information qui cherchent à isoler le journaliste comme seul « responsable» de l'écriture de l'article ou comme le maillon fort de la chaîne de production. Sous cet angle, l'analyse du système journalistique relève de ce qu'il conviendrait d'appeler aujourd'hui une « ethnographie de la communication » et particulièrement si on y inclut les aspects non verbaux tels que les gestes professionnels (Agostinelli, 2003, a).

Nous allons présenter à travers la définition des divers concepts de médiation, de coopération et de contextualisation comment se structure l'élaboration de l'information journalistique dans une organisation. Nous allons voir comment les communications instaurent les relations et les interactions entre les acteurs. Cette 


\section{Expériences}

présentation est également illustrée par les exemples de terrain que nous avons recueillis grâce à une observation participante au sein d'un organisme de presse régionale.

\section{L'information journalistique entre processus de communication et de médiation \\ L'information journalistique comme un processus de communication}

$\mathrm{Au}$ sein des rédactions de presse écrite les communications aussi bien, verbales, qu'écrites ou même médiées, sont continuellement présentes et surtout nécessaires pour construire en continu le journal papier. Les discussions avec les collègues au sein des réunions de rédaction, les interactions avec le rédacteur en chef durant l'écriture du papier sont autant de communications nécessaires à l'élaboration du papier final. Nous pensons comme Jean-Luc Bouillon (2003) que: « $L a$ communication, «les communications", plus exactement, semblent faire référence à une sorte "d'éther" dans lequel baigneraient actions individuelles et collectives, organisations et rapports sociaux. Elles sont tellement omniprésentes qu'elles en deviennent diffuses, peut-être trop insaisissables pour être analysées en tant que telles. Il nous semble pourtant essentiel de focaliser notre attention sur ces phénomènes polysémiques » (Bouillon, 2003, p. 8). Pour les étudier, nous avons analysé les acteurs en situation, c'est-à-dire au sein des rédactions et au moment de l'élaboration de l'article. Nous avons observé les différents acteurs du système journalistique (rédacteur, secrétaire de rédaction, responsable de la pagination, rédacteur en chef, photographes...) pour comprendre leurs rôles respectifs et leur impact sur le journal. Nous pensons que l'analyse des situations de communication au quotidien est nécessaire pour comprendre comment les relations et les interactions entre les acteurs, voire même les médiations structurent le processus de production de l'information, c'est-à-dire le geste professionnel d'écriture de l'article de presse (Bouillon, 2003). Comme pour la photographie de presse (David, 2002) nous pensons que le geste professionnel d'écriture de l'information est "un sens négocié s'élaborant dans l'entre des échanges formels et informels » (David, 2002, p.72) entre les acteurs. De plus, ces gestes permettent de rendre "compte de l'histoire d'une construction où les objets ne sont pas "les buts [du] collectif, [mais] en sont les conditions »(Serres, 1983, p.111)» (David, 2002, p. 73).

Lorsqu'on s'attache à comprendre comment les interactions et les collaborations entre les rédacteurs produisent une information, et comment les relations avec les lecteurs médiées par le journal 
La communication organisationnelle au sein d'une entreprise de presse écrite

influencent les articles futurs des rédacteurs, il est tout d'abord nécessaire de comprendre comment on passe d'une donnée de terrain à une information. Pour cela le rédacteur élabore un processus cognitif, permettant le passage d'une donnée brute à une information, puis à une connaissance. Au départ la donnée est "un fait brut codé » (Le Bœuf \& al, 2000, p. 15) que le rédacteur va chercher sur le terrain par le biais de ses observations, ses reportages, ses interviews etc. Cette donnée de terrain permet au rédacteur d'élaborer un article, c'est-à-dire une construction de sens nécessaire à une interprétation contextuelle de la donnée. Cette construction donne du sens à la donnée de terrain et la transforme en information. Les journalistes en rédigeant l'article font ainsi de l'information préconstruite. L'information est alors entendue comme "le traitement rationnel, et l'état qui en résulte, de données objectives, matérielles et symboliques, manipulables et stockables dans des documents physiques» (Linard, 2001, p. 212). "L'information est une quantité de probabilité ou de variation d'évènement, transcrite en une organisation syntaxique de symboles purement formels entraînant des différences de traitement purement physiques dans un système matériel. Ce système inerte est par principe insensible à son environnement (sauf pour ces conditions mécaniques de fonctionnement). C'est un outil d'exécution, indifférent par principe aux significations des symboles utilisés et aux résultats produits " (Linard, 1994, p. 120). Elle est aussi, comme la définit Edgar Morin, "une quantité de données transférées d'un individu à un autre. Elle instruit en apportant une connaissance. Elle tend donc à réduire sinon à supprimer l'incertitude» (Morin, 1990, p. 145). En journalisme, cette information est indexicalisée collectivement et permet l'élaboration d'un sens commun entre les divers acteurs du système de rédaction. Une fois publiée dans le journal, c'est-à-dire structurée et organisée socialement, cette information devient alors une connaissance pour le lecteur. Elle relève de l'accountabilité et peut être définie: "chez un individu, [comme] l'état subjectif interne de ces données produites par assimilation et auto-transformation mentale» (Linard, 2001, p. 212). "La connaissance est une organisation syntactico-semantico-pragmatique d'un système vivant, bio-psycho-sociologique, directement intéressé aux résultats de son activité pour sa survie et sa satisfaction. Largement qualitative et formalisée seulement en partie, la connaissance est auto-générée par un système hyper sensible à son contexte qui évolue et produit des interprétations de décisions et des actions en fonction de ses interactions (de son expérience) avec le milieu dont il dépend. C'est cette situation d'interdépendance avec son milieu qui rend les 


\section{Expériences}

résultats de son activité plus ou moins intéressants pour le système et lui fournit des critères sélectifs et cohérents de valeurs, de signification et de jugement» (Linard, 1994, p. 120). Dans cette recherche nous considérons les connaissances non pas comme des acquis individuels qui résideraient en tant que tels en mémoire de chacun, mais comme une action supposant la participation d'un individu à un ensemble contextualisé de pratiques (Agostinelli, 2003, b). Nous envisageons ainsi les connaissances comme "l'expression d'un processus d'interaction complexe qui met en relation un individu, que l'on pense apte à saisir les régularités de la situation qu'il traverse et le réel fortement socialisé dans lequel il navigue et doit se faire entendre » (Agostinelli, 2003, b, p. 133). Tout en construisant cette connaissance, l'individu apprend et élabore une "communauté de pratique, d'usages et d'échanges " (Agostinelli, 2003, b, p. 133) dans laquelle il va mettre à l'épreuve ses connaissances, en connaître de nouvelles et créer une situation de communication, avec des règles et des contraintes contextualisées. L'activité des journalistes, pour passer de la donnée brute de terrain à l'information pour le lecteur nécessite des « pratiques routinisées » ou des genres de «l'art décrire » c'est-à-dire l'élaboration d'un "geste professionnel d'écriture ».

Pour nous, " un acte social est effectué par un individu en fonction de la situation totale dans laquelle il s'inscrit, en fonction des influences reçues des conduites des autres et de ce qu'il pense provoquer chez les autres étant donné son aptitude à évoquer en lui les réactions des autres à son action" (Mucchielli, 1998, p. 13). Ainsi, lorsque les journalistes élaborent le geste professionnel, c'est-à-dire leur manière de faire relevant de processus particuliers et d'une réglementation propre à chaque organisation (processus d'écriture, d'interaction...) ils tentent en réalité de provoquer chez le lecteur une modification de sa représentation sociale de la réalité : "le sens d'un geste accompli par un organisme se trouve dans la réponse de l'autre organisme à ceux qu'aurait été l'achèvement de l'acte du premier organisme que ce geste annonce et indique » (Mead, 1934, p. 145). Le geste d'écriture du rédacteur, par exemple, ne prend tout son sens que dans le geste de lecture des lecteurs, qui par retour de courrier, lui indique l'adéquation ou non à ses attentes. On voit ainsi que le geste du journaliste n'existe que par la réaction du geste des personnes avec qui il est en interaction. Ces interactions et relations entre les acteurs du système se font grâce au processus de communication qu'élaborent les acteurs. Ces communications peuvent être directes, comme c'est le cas entre le rédacteur et le secrétaire de rédaction au sein du journal lors de la 
La communication organisationnelle au sein d'une entreprise de

presse écrite

mise en forme du papier; ou encore, avec le rédacteur en chef au cours de la décision de l'angle d'attaque. Elles peuvent être aussi indirectes comme c'est le cas avec les lecteurs. Les journalistes rédacteurs ne communiquent pas directement avec leurs lecteurs, ils rentrent en relation avec eux grâce au journal et reçoivent un feedback par l'intermédiaire du courrier des lecteurs. Toutes ces communications influencent directement le geste professionnel d'écriture du journaliste car il écrit pour un lectorat spécifique dans une organisation particulière.

L'information journalistique nécessite pour émerger un modelage spécifique. Elle est considérée "comme une donnée immanente à transmettre le plus fidèlement possible: elle est interpellée, reformatée, saisie, traduite par de nombreux acteurs aux fonctions tantôt transformatrices, interprétatives ou normatives. C'est dire que l'information résulte et s'accompagne sans cesse d'une série d'interventions tout autant intellectuelle, politique que technique, qu'elle est produite et utilisée dans et sous certaines conditions » (Guyot, 2004, p. 1). Cette transformation est le résultat de processus cognitifs des journalistes dans un contexte particulier, celui d'un organisme de presse régionale pour un lectorat régional. Le rédacteur qui rédige seul son papier, face à son ordinateur va faire émerger une information qu'il va devoir confronter d'une part, à son chef de service et à son rédacteur en chef, pour l'angle d'attaque et la ligne éditoriale du journal, ensuite au terrain pour la validité et au secrétaire de rédaction pour la forme et le fond. Sa donnée de départ se construit donc en fonction de normes d'écriture établies par l'organisation et devient une information partagée et située.

En journalisme, la connaissance est une structuration de l'information que le journaliste transmet à l'aide du journal. Son intention de communication est d'informer le lecteur en lui donnant une préconstruction de l'information, issue de la donnée de terrain, afin qu'il se construise lui-même sa propre connaissance. C'est grâce à l'interaction avec les journalistes médiés par le journal que le lecteur peut se construire une vision du monde et de la réalité qui l'entoure. Les rédacteurs par leur article diffusent ainsi un prêt à penser d'information structurée à partir de données de terrain. Cette perception n'est évidemment pas objective car elle préconstruit un sens, celui perçu par les rédacteurs.

On voit donc déjà que la mise en place des processus de communication écrite, au sein des rédactions journalistiques, nécessite des ajustements, des adaptations pour élaborer collectivement de 


\section{Expériences}

nouvelles connaissances. Cette construction est liée à la capacité qu'ont les individus, ici les journalistes, à mobiliser ensemble leurs connaissances dans une situation particulière de travail collaboratif (Bouillon, 1999).

Nous venons de voir que les processus de communication qui s'établissent entre les acteurs provoquent des interactions et des médiations. Les processus de communication au sein des organismes de presse sont donc couplés de processus d'interaction et plus particulièrement de médiation. Dans la partie qui suit, nous définissons ce que nous entendons par interaction et médiation.

\section{L'information journalistique comme processus de médiation}

La notion de médiation est perçue dans ce travail comme le fruit d'interactions sociales médiées par un outil, ici, le journal papier. Les sujets sont des êtres incarnés, auto actifs, qui régulent leur action en fonction de leur intention et résultat. Ils sont socialement organisés et leur intention est motivée en fonction des besoins qu'ils développent. L'interaction entre sujets et objets n'est donc ni neutre, ni mécanique : c'est une quête intentionnelle intéressée d'objets par des sujets au sein d'un groupe social (Vygotski, 1997). "Les activités humaines sont donc socialement médiatisées, c'est-à-dire elles sont instrumentées, structurées et transformées par des "procédures», des "outils» socialement construits » (Agostinelli, 2003, p. 136). L'activité est en rapport direct avec la notion de médiation (Rivière, 1990), qui permet par l'intermédiaire de l'outil de transformer l'activité en une représentation particulière (Agostinelli, 2003, b) : "les activités ne sont plus seulement présentes dans leur seule exécution. Elles existent en quelque sorte indépendamment d'elle, dans les outils qui les représentent, et par là même, signifient» (Schneuwly, 1986, p. 7). La médiation est perçue comme le lien conçu entre le monde externe et la conscience. Grâce aux interactions que l'individu entretient avec cet environnement, il développe ses fonctions psychiques supérieures, en s'appropriant la culture qui l'entoure. Pour Vygotski, les fonctions psychiques supérieures sont « unies par une caractéristique commune, celle d'être des processus médiatisés, c'est-à-dire d'inclure dans leur structure, en tant que partie centrale et essentielle du processus dans son ensemble, l'emploi du signe comme moyen fondamental d'orientation et de maîtrise des processus psychiques » (Vygotski, 1997, p. 199). Il en est ainsi en journalisme ; le rédacteur fait évoluer son article en fonction de l'environnement qui l'entoure, mais aussi en fonction des interactions qu'il entretient avec lui. Si durant une manifestation, par exemple, il rencontre un interlocuteur très intéressant il décidera, alors, de faire un papier interview plutôt qu'un 


\section{La communication organisationnelle au sein d'une entreprise de \\ presse écrite}

papier compte rendu. Sa perception de la manifestation sera orientée par la représentation qu'en fait son interlocuteur. Grâce à ces interactions le journaliste modifie, d'une part la mise en forme des données de terrain et donc l'angle d'attaque, il ne traite pas le sujet de la même manière; d'autre part, sa représentation mais aussi par la médiation via le journal celle de ses lecteurs. De ce fait, son activité psychique évolue au cours de la construction de l'information à travers l'article, grâce aux interactions et médiations qu'il entretient avec ses collaborateurs, ses sources, ses lecteurs, mais aussi avec la situation dans laquelle il évolue. L'article de presse élaboré par le système journalistique est considéré comme une médiation de l'information, une réorganisation de la connaissance des lecteurs et donc de leur mode de représentation de la réalité. Ces processus de médiation mettent en place des processus inconscients qui donnent les clés nécessaires au lecteur pour avoir une perception orientée de la réalité sociale. Ainsi l'information médiée par le journal sert de catalyseur pour modifier les représentations des lecteurs et leur donner une perception préconstruite de la réalité qui les entoure. Un des exemples les plus flagrants est l'émulation que provoque l'annonce d'un évènement historique par les médias. Les lecteurs ou les spectateurs croient à l'évènement du moment qui passe dans le journal ou à la télévision, c'est-à-dire qu'il est médiatisé. Or, il oublie souvent que cette médiatisation est avant tout une médiation, c'est-à-dire une construction d'acteurs en interaction grâce à un outil : le journal. Cette construction est située, orientée et contextualisée en fonction du contexte dans lequel elle est immergée (contexte politique, économique, social...), mais aussi en fonction du journal dans lequel elle est éditée. Cette information est donc le résultat d'une perception particulière d'individus: les journalistes et non la perception universelle de la réalité. Cette construction de la réalité est le résultat d'une action située et partagée par un collectif d'individus, les journalistes, dans un contexte spécifique.

On vient de voir que le geste professionnel d'écriture de l'article des journalistes de presse écrite, et plus particulièrement dans notre cas de presse régionale, est le résultat de plusieurs processus. Des processus de communication qui permettent aux acteurs du système d'être en interaction et de construire ensemble un article, mais aussi des processus de médiation qui, via le journal, favorisent une construction commune de la réalité. Nous constatons donc que l'article 


\section{Expériences}

journalistique n'est plus perçu ${ }^{1}$ uniquement comme un outil de médiatisation de l'information, mais bien comme un construit social où chaque acteur du système donne son point de vue. Cette construction de l'article passe par des processus de communication mais aussi par des processus collectifs et coopératifs de travail.

Nous montrons maintenant comment les journalistes élaborent une information grâce à ces processus collectifs et coopératifs, dans un contexte spécifique.

\section{L'information journalistique comme le résultat d'un travail collaboratif dans un contexte spécifique}

\section{Le journalisme un travail collectif et coopératif situé}

Aujourd'hui l'ampleur des études sur le travail collectif et coopératif en ergonomie laisse à se demander, comment on a pu penser l'activité comme purement individuelle et la cognition comme un processus interne. Pour nous, comme pour Jacques Theureau (2003), les activités individuelles sont articulées, voire imbriquées entre elles. Comprendre l'analyse du travail journalistique nécessite d'étudier le lien entre l'activité des opérateurs (rédacteurs, secrétaires de rédaction, responsables de la pagination, etc.), les caractéristiques de leur situation, leur bien être et leur épanouissement, en résumé de prendre en compte l'articulation entre l'activité individuelle et l'activité collective. Ceci nous permet d'entendre la communication humaine comme une activité collective, en ceci qu'elle est distribuée entre plusieurs personnes et gérée à travers leurs interactions (Sperber, 2001). La presse écrite peut être perçue comme un dispositif au sein duquel il est nécessaire de développer une action collective productive à partir de la coordination de tâches individuelles (Bouillon, 2003). En effet, chaque acteur du système possède un rôle respectif, bien déterminé dont l'objectif est de s'accorder avec le travail des autres pour constituer au quotidien le journal. La production quotidienne du journal recouvre l'activité d'élaboration d'un produit précédant sa

1 De nombreux modèles se sont intéressés au cours des 1960 à 1970 au développement des mass media. Leur objectif était de comprendre les relations qui s'établissent entre les sources de production, les entreprises de production, les médias et le public. Ces divers modèles d'étude sur les médias, allant de celui de Schaeffer (1971) qui s'intéresse à la «culture de masse ", à celui de Moles (1967) qui par une approche socioculturelle étudie les industries de production et de diffusion comme des systèmes d'information, traitent peu du contexte d'immersion des interactions liées au travail collaboratif des journalistes. Des auteurs récents, comme Charaudeau (1999) par exemple s'intéressent davantage à la médiatisation de l'information qu'à la médiation. 
La communication organisationnelle au sein d'une entreprise de presse écrite

réalisation, où chaque acteur a la charge d'une tâche spécifique (Bouzon, 2003). Par exemple, les responsables de la pagination qui montent le squelette du journal, permettent aussi aux rédacteurs de rédiger le papier, aux secrétaires de rédaction de mettre en forme, aux photographes d'illustrer etc. Ces actions multiformes reposent sur la coopération entre acteurs, au sein d'une équipe de rédaction.

Le journal est avant tout une organisation, au-delà de laquelle chaque acteur agit en fonction de sa situation et de ses intérêts, dans le but de réaliser une action collective qui est la production d'un quotidien. Toutefois l'observation de terrain que nous avons réalisée nous a permis de comprendre qu'il faut s'intéresser à l'action collective dans le processus de production de l'information. Pour définir l'activité collective, on peut se référer à une formulation inspirée de Jacques Leplat (1993): "il y a activité collective chaque fois que l'exécution d'une tâche entraîne l'intervention coordonnée de plusieurs opérateurs. "Entraîne »: il s'agit d'une activité effective. "Coordonnée »: les opérations sont en interactions, c'est-à-dire que leurs activités dépendent les unes des autres. Une activité collective n'est pas une collection d'activités individuelles sur des tâches indépendantes mais une activité où des opérateurs réalisent conjointement la même tâche, dans un même lieu, où éventuellement dans un lieu différent »(Leplat, 1993, p. 10). Dans sa conception du travail collectif cet auteur avance deux aspects : d'une part, les acteurs impliqués dans la même tâche de travail se partagent le même but et les mêmes conditions de travail; d'autre part, leurs activités sont interdépendantes. Dans un journal quotidien, les journalistes (rédacteurs, photographes, secrétaires de rédaction documentalistes...) partagent leur processus de travail dans le but de construire en commun : le journal papier. Les outils informatiques de mise en page sont, par exemple, des processus partagés entre les acteurs afin d'harmoniser les tâches individuelles pour rendre un journal papier cohérent et structuré. On peut, ainsi, considérer que les diverses tâches mises en place au sein des rédactions relèvent bien d'un travail collectif des acteurs, voire même au-delà d'un travail coopératif, car il y a une dépendance mutuelle des actions. Nous avons pu observer qu'au niveau de l'organisation du travail, l'espace est organisé tantôt en espace personnalisé pour permettre l'autonomie de chaque acteur, tantôt en espace collectif pour construire le journal. Par exemple, nous avons pu remarquer que dans certaines rédactions les postes de travail sont personnalisés (photographies, bibelots, étiquettes avec le nom sur les casiers de rangement...), auquel s'ajoute l'utilisation d'un «password» personnel pour entrer dans le système informatique. 


\section{Expériences}

Toutefois, cette personnalisation des postes est organisée de telle sorte que la coopération soit possible. Les espaces de travail sont assez proches les uns des autres, favorisant ainsi la proximité et l'échange, le système informatique permet la libre navigation des rédacteurs au sein du journal en cours d'élaboration. Le décloisonnement de l'espace de travail favorise ainsi les interactions et les processus de communication nécessaires à la rédaction des articles. L'action collective est donc une action organisée, c'est-à-dire naturellement structurée autour d'un certain mode d'organisation (Friedberg, 1993). Mais le travail des journalistes en rédaction va plus loin qu'un simple travail collectif, il est un travail coopératif, c'est-à-dire que les acteurs mettent en place des processus pour élaborer une activité commune. La coopération entre acteurs est le processus par lequel des opérateurs participent à une activité commune. Cette coopération est entendue à partir du moment, où d'une manière imposée par la hiérarchie ou prescrite par la volonté des acteurs eux-mêmes, il y a un but commun ou interférence entre les buts de chaque acteur (Barthe \& Quéinnec, 1999).

L'étude de la construction de l'information peut donc s'analyser par l'observation des interactions et des médiations, mais aussi par l'examen des comportements collectifs, dont l'approche se situe à la croisée du social et du cognitif. Celle-ci traite de problèmes voisins sous des appellations diverses, telles que «l'action située», la « cognition située », et la « cognition distribuée » qui expriment toutes la même idée de médiation entre l'action et l'environnement (Conein, 1994). Le concept «action située » étudié par Lucy Suchman (1987) indique que "l'action ne se limite pas à l'interaction, mais est aussi orientée vers un but et dépend des moyens et des circonstances $d u$ moment» (Bouzon, 2003, p. 5). Elle devient «cognition située» lorsque le chercheur se donne comme projet de rendre explicites les supports informationnels qui ancrent l'action dans son environnement immédiat (Conein, 1994). Ce concept d'action située nous permet de comprendre la production de l'information par le système journalistique, comme une action collective des divers acteurs de la situation de communication, en cours d'action, pour mettre en avant que les rédactions s'organisent en système d'interactions contextualisées où les journalistes construisent des représentations collectives de l'information. Cette perception est assez visible au cours des réunions de rédaction. L'hypothèse connexionniste sousjacente pose que l'intelligence est la propriété émergente du collectif et que le fonctionnement d'une équipe dépend du réseau de 
La communication organisationnelle au sein d'une entreprise de presse écrite

connexions entre les acteurs concernés, pour construire collectivement le sens de la situation (Bouzon, 2003).

$\mathrm{Au}$ sein de cette organisation, la capacité à constituer un collectif, à partager un même contexte de mobilisation des connaissances est essentielle (Bouillon, 2003). Dès lors, notre objet de recherche s'attache à la compréhension des comportements humains et de leurs interactions dans un contexte d'action (Friedberg, 1993). Ce contexte d'action est spécifique au moment de l'élaboration de l'article. Il est directement lié aux acteurs de la situation, à l'organisation et doit être envisagé par ses aspects indexical et réflexif.

\section{L'information une construction en situation}

Nous partons du principe que l'information journalistique est également construite en fonction de la situation organisationnelle dans laquelle elle est immergée. On n'écrit pas ainsi de la même manière dans un quotidien régional comme Midi Libre (généraliste), que dans un journal plus "orienté, engagé » comme le Canard Enchaîné... Au sujet de la politique par exemple, les titres du Canard Enchaîné proclament "Ségolène court à la dégelée royale », ou "Tempête sur le couple Sarko-Balkany », alors que Midi Libre reste plus réservé : "l'emploi des jeunes cible dans l'offensive Villepin » ou «Sarkozy au chevet des usagers du Nice-Lyon» (De Ceglie, 2006). Ainsi, une situation d'information est "un moment de l'activité, un espace-temps totalement contextualisé par elle. $Y$ sont réunis une intention, un contexte et une temporalité » (Guyot, 2004, p. 2). Nous entendons par contexte un environnement local, organisé socialement par des actions concertées dont l'intérêt est en rapport avec le projet des acteurs (Agostinelli, 2001, b). Le contexte situationnel devient donc un contexte indexical et récursif, c'est-à-dire qu'il permet par sa récursivité une (ré)information et une remise en question de la compréhension des individus, permettant une distanciation par rapport à la situation (Agostinelli, 2001, a). Le contexte est donc constitué par l'activité des acteurs en situation qui, en retour, les informent d'une façon active. Dans le journalisme, par exemple, le contexte rédactionnel est perpétuellement en évolution. Tout d'abord, parce qu'au sein des rédactions les va-et-vient continus des différents journalistes multiplient les interactions entre les acteurs, ce qui peut influencer l'écriture de l'article. De plus, l'arrivée incessante des nouvelles informations de l'AFP' ${ }^{2}$, fait également évoluer les données, les points de vue et par conséquent le contexte d'immersion. Lors

${ }^{2}$ AFP : Agence France Presse 


\section{Expériences}

d'une de nos observations au sein d'une rédaction, un « scoop », c'està-dire une information fortement indexicalisée, car elle est partagée par le lecteur et dépasse donc le seul journaliste qui la diffuse, arriva vers vingt-trois heures juste avant l'impression du journal. Cette nouvelle perturba en quelques secondes, non seulement la rédaction concernée, mais également les autres qui durent revoir l'intégralité de leur mise en page, les coupures à effectuer... on voit ainsi que lors du geste professionnel d'écriture en rédaction, le contexte est constamment en évolution. Il y a donc une même construction du monde entre les journalistes et le social, puisque l'information est construite pour le lecteur qui dès le lendemain achète le journal. La contextualisation des actions des rédacteurs, c'est-à-dire l'adaptation en continu des données à l'évolution du social, au sein du système journalistique, se référent à un ensemble d'actions concerté localement et socialement organisé (Garfinkel, 1967). Elle est construite avec un caractère indexical récursif dans une situation de travail où se couplent des idées d'équilibre et de déséquilibre, de stabilité et d'instabilité, de dynamique et de constance (Agostinelli, 2004).

\section{Conclusion}

$\mathrm{Au}$ cours de cet article nous avons pu voir que le geste professionnel des journalistes relève de processus complexes de communication, d'interaction, de médiation et de travail collectif. Ces processus émergent dans un contexte spécifique qui les modifient et qu'ils modifient en retour, permettant une adaptation permanente de l'information journalistique à la situation organisationnelle dans laquelle elle est élaborée. Nous avons également vu que l'information journalistique est le résultat d'une activité professionnelle collaborative voire coopérative entre les différents acteurs de l'organisation. Ainsi, nous avons pu montrer qu'étudier un organisme de presse pouvait se faire sous l'angle communicationnel et interactionnel en s'attachant non pas uniquement à la médiatisation de l'information, comme c'est souvent le cas dans les recherches en journalisme, mais plus au processus de médiation, d'interaction et de communication que mettent en place les acteurs de l'organisation pour construire collectivement une perception de la réalité, à un moment donné.

\section{Bibliographie}

Agostinelli, S., "Contexte et système complexe artefactuel », in: Réseaux d'information et non linéarité. L. Vieira, \& N., W., Pinède, 
La communication organisationnelle au sein d'une entreprise de presse écrite

(eds.). Bordeaux, Maison des Sciences de l'Homme d'Aquitaine, 2001, a, p.133-144.

Agostinelli, S., Voies de recherche en Sciences de l'Information et de la Communication : Le rôle des artefacts et des organisations sociales dans la communication des connaissances. Habilitation à Diriger des Recherches, Université de Rennes II, 2001, b.

Agostinelli, S., « Décrire un processus métier, n'est pas seulement une question de méthode mais probablement aussi, une façon de regarder les gestes professionnels ». In: Pecquet, P., (eds.) Manager l'entreprise virtuelle des processus métiers pour quoi faire? Montpellier I, Cdrom, 2003, a.

Agostinelli, S., Les nouveaux outils de la communication des savoirs. Paris, L'Harmattan, 2003, b.

Agostinelli, S., «Le sens des choses ou le besoin de sens ». In: Les vallées : sens, territoires et signes. GREC/O, 25, Bordeaux, Maison des Sciences de l'Homme d'Aquitaine, 2004, p. 83-95.

Barthe, B., \& Quéinnec, Y., «Terminologie et perspectives d'analyse du travail collectif en ergonomie », in: L'Année Psychologique, 99. Paris, A.Colin, 1999, p. 663-686.

Bouillon, J.-L., Vers une approche communicationnelle de la gestion des connaissances. Thèse pour le doctorat en SIC, Université de Toulouse le Mirail / LERASS, 1999.

Bouillon, J.-L., «Pour une approche communicationnelle des processus de rationalisation cognitive des organisations: contours, enjeux et perspectives. Supports, dispositifs et discours médiatiques à heure de l'internationalisation "), in: Colloque bilatéral francoroumain CIFSIC. Université de Bucarest, 28 juin- 3juillet 2003. Consulté sur : http://archivessic.ccsd.cnrs.fr

Bouzon, A., "Coopération et communication dans les projets de conception collective. Supports, dispositifs et discours médiatiques à heure de l'internationalisation ", in: Colloque bilatéral francoroumain CIFSIC. Université de Bucarest, 28 juin- 3 juillet 2003. Consulté sur : http://archivessic.ccsd.cnrs.fr

Bouzon, A., \& Meyer, V., «Introduction ». In : Bouzon, A., \& Meyer, V. (sous la dir.), La communication organisationnelle en question : méthodes et méthodologies. Paris, L'Harmattan, 2006.

Caron, C., «De l'appropriation inachevée du genre en communication organisationnelle ». In: Communication \& Organisation, $\mathrm{n}^{\circ} 25$. Bordeaux, Maison des Sciences de l'Homme d'Aquitaine, 2004, p. 180-196. 


\section{Expériences}

Charaudeau, P., «La médiatisation de l'espace public », in: Etudes de communication. La médiatisation des problèmes publics, $\mathrm{n}^{\circ} 22$. Paris, Séminaire annuel CARISM/IFP, 1999, p. 74.

Conein, B., "Introduction au numéro travail et cognition», in Sociologie du travail, numéro spécial Travail et cognition, XXXVI, 4. Paris, Masson, 1994, p. 419-425.

David B., "La photographie de presse dans les cadres du chercheur », in: Etudes de communication, $n^{\circ} 25$. Lille 3, Université Charles de Gaulle, 2002, p. 71-85.

David B., " Une approche ethnographique des entreprises de presse », in: Acte des journées Org\&Co: Organisation communicante et communication organisante. Castres, 2004.

De Ceglie, A., «L'ethnométhodologie dans l'analyse d'un processus collectif de création d'information », in: Bouzon, A. \& Meyer, V. (sous la dir.), La communication organisationnelle en question: Méthodes et Méthodologies. Paris, L'Harmattan, 2006, p. 167-187.

Friedberg, E., Le pouvoir et la règle - dynamique de l'action organisée. Paris, Seuil, collection Sociologie, 1993.

Garfinkel, H., Studies in ethnomethodology. Cambridge : PolityPress, 1967.

Guyot, B., "Elément pour une approche informationnelle dans les organisations ", in : Science de la société, $\mathrm{n}^{\circ}$ 63. Toulouse : Presse Universitaire du Mirail, 2004, p.11-25. Consulté sur : http://archivessic.ccsd.cnrs.fr

Hutchins, E., Cognition in the Wild. Cambridge, MA, MIT Press, 1995.

Lave, J., Cognition in Practice. Cambridge, UK: Cambridge, University Press, 1988.

Le Boeuf, C., Drouillière, L., \& Rivière, L., Introduction à la communication. Paris, Foucher, 2000.

Leplat, J., "Ergonomie et activités collectives », in: F. Six \& X. Vaxevanoglou (Eds.), les aspects collectifs du travail, Toulouse, Octarès, 1993, p. 7-27.

Linard, M., «Vers un sujet narratif de la connaissance dans les modélisations de l'apprentissage », in Intellectica, $n^{\circ} 19$. Paris, ARCCNRS, 1994, p. 117-165.

Linard, M., "Concevoir des environnements pour apprendre: l'activité humaine, cadre organisateur de l'interactivité technique », in Delozanne, E., et Jacoboni, P., « interaction homme-machine pour la 
La communication organisationnelle au sein d'une entreprise de presse écrite

formation et l'apprentissage humain ", Sciences et techniques éducatives, vol. 8, n³-4. Paris, Hermès Science, 2001, p. 211-238.

Le Moënne, C., "Considération sur les méthodes de recherche en communications organisationnelles ", in Bouzon, A., et Meyer, V. (sous la dir.), La communication organisationnelle en question: méthodes et méthodologies. Paris, L'Harmattan, 2006, p. 15-30.

Mead, G.H., Mind Self and Society. Chicago, University of Chicago Press. Trad. Fr.: l'Esprit, le Soi et la Société. Paris, PUF, 1934/1963.

Moles, A., \& Oulif, J-M., « Le troisième homme, vulgarisation scientifique et radio », in : Diogène, $n^{\circ} 58$. Paris, PUF, 1967.

Morin, E., Introduction à la pensée complexe. Paris, ESF, 1990.

Mucchielli, A., Les Sciences de l'information et de la communication. Paris : Hachette, 1998.

Rivière, A., La psychologie de Vygostki : «Psychologie et Sciences Humaines ». Sprimont (Belgique), Edition P. Mardaga, 1990.

Salembier, P., \& Zouinar, M., Intelligibilité mutuelle et contexte partagé, Inspirations conceptuelles et réduction technologiques, in Revue @ ctivités, 1(2), 2004, p. 64-85.

Schaeffer, P., Machine à communiquer. Paris, Seuil, 1971.

Schneuwly, B., "Les capacités humaines sont des constructions sociales. Essai sur la théorie de Vygotski », in: European Journal of Psychologie of Education, 1. Paris, Masson, 1986, p. 5-16.

Serres, M., Rome. Le livre des fondations. Paris, Grasset, 1983.

Sperber, D., «L'individuel sous l'influence du collectif», in : $L a$ Recherche, 344, 2001, p. 32-35.

Suchman, L., Plans and situated actions: The problem of humanmachine communication. New York, Cambridge University Press, 1987.

Thereau, J., «Introduction à la session $1:$ une question de recherche en analyse de travail essentiel pour l'ergonomie, celle de l'analyse de l'articulation entre activité individuelle et activité collective », in $38^{e}$ Congrès de la SELF, Paris, 2003.

Vygotski, L.S., Pensée et Langage. Paris, La Dispute, 1997. 